\title{
Electrons rapides dans un plasma créé par laser
}

\author{
C. Rousseaux ( $\left.{ }^{1}\right)$, F. Amiranoff $\left({ }^{1}\right)$, C. Labaune ( $\left.{ }^{1}\right)$, B. Mabille ( ${ }^{2}$ ) et G. Matthieussent $\left({ }^{3}\right)$ \\ GRECO Interaction Laser Matière, Centre National de la Recherche Scientifique, Ecole Polytechnique, \\ 91128 Palaiseau Cedex, France \\ (1) L.U.L.I., Ecole Polytechnique, 91128 Palaiseau Cedex, France \\ (2) C.P.T., Ecole Polytechnique, 91128 Palaiseau Cedex, France \\ (3) L.P.G.P., Orsay, France
}

(Reçu le 15 octobre 1987, accepté le 20 mai 1988)

\begin{abstract}
Résumé. - Nous présentons ici des spectres énergétiques des électrons créés au cours de l'explosion de feuilles minces par laser à haut flux à $\lambda=1,06 \mu \mathrm{m}, \lambda=0,53 \mu \mathrm{m}$ et à $\lambda=0,26 \mu \mathrm{m}$. Nous exposons les diagrammes angulaires électroniques à $\lambda=0,26 \mu \mathrm{m}$ pour différentes énergies, caractérisés (au-dessus de $150 \mathrm{keV}$ ) par une structure piquée le long de l'axe laser. Ces électrons peuvent être en partie expliqués par leur accélération dans l'onde de plasma issue de l'instabilité Raman arrière.
\end{abstract}

\begin{abstract}
High energy electrons (up to $250 \mathrm{keV}$ ) emitted from laser irradiated thin foils at high intensities have been measured at three laser wavelengths $(1.06,0.53$ and $0.26 \mu \mathrm{m})$ and at different angles from the target normal. The energy spectra show a preferential emission of the highest energy electrons $(>150 \mathrm{keV})$ in the direction of the incident laser beam. The plasma waves created by the Raman backward scattering are a possible generation mechanism for the forward acceleration of these electrons.
\end{abstract}

\section{Introduction.}

Nous présentons une étude des électrons rapides générés dans un plasma créé par laser. Elle est motivée par deux pôles en particulier. En premier lieu, dans le cadre de la fusion inertielle par laser, on s'efforce de comprimer puis de chauffer le plus possible de la matière fusible ; pendant la phase de compression où le laser ablate la cible, il interagit avec ce plasma ; de cette interaction au flux nécessairement élevé naissent des instabilités paramétriques qui peuvent, dans le pire des cas - diffusion Brillouin [1] - renvoyer presque toute l'énergie incidente vers l'arrière. Certaines de ces instabilités, telles que la diffusion Raman stimulée [2] ou la décomposition en deux plasmons [3], créent des ondes électrostatiques de fortes amplitudes (les ondes de plasma) dans lesquelles une partie des électrons sont puissamment accélérés. Ces électrons très énergétiques, lorsqu'ils précèdent le front d'ablation, déposent leur énergie dans le cœur de la cible, encore froid; ceci réduit considérablement l'efficacité de la compression et par suite les taux de combustion espérés.

En second lieu, la détection de ces électrons met en évidence l'existence dans de tels plasmas de champs électriques longitudinaux élevés. Ceci rentre dans le cadre de l'étude d'accélération de particules chargées de haute énergie dans les plasmas. L'ordre de grandeur de ces champs est donné comme suit : l'observation d'électrons de plus de $250 \mathrm{keV}$ sur une longueur d'accélération d'au plus $100 \mu \mathrm{m}$ (longueur du plasma) entraîne l'existence d'un champ électrique d'au moins $2,5 \mathrm{GV} / \mathrm{m}$. Outre la compréhension du mécanisme d'accélération des particules dans de tels champs, la maîtrise de leur création par l'interaction laser-matière pourrait contribuer à l'élaboration de futurs accélérateurs.

Nous nous sommes attachés ici à caractériser la distribution tant angulaire qu'énergétique de ces électrons, essentiellement à $\lambda=0,26 \mu \mathrm{m}$.

\section{Schéma et caractéristiques de l'expérience.}

Le schéma de principe se trouve en figure 1. Le laser est focalisé sur cible de terphane $\left(\mathrm{C}_{8} \mathrm{H}_{10} \mathrm{O}_{4}\right)$ d'épaisseur $1,5 \mu \mathrm{m}$, en une tache focale de $50 \mu \mathrm{m}$ à miénergie, à l'aide d'une lentille de focale $250 \mathrm{~mm}$ ouverte à $f / 2,7$. Le plan de la cible est perpendiculaire à l'axe du faisceau. Le laser dopé au néodyme 


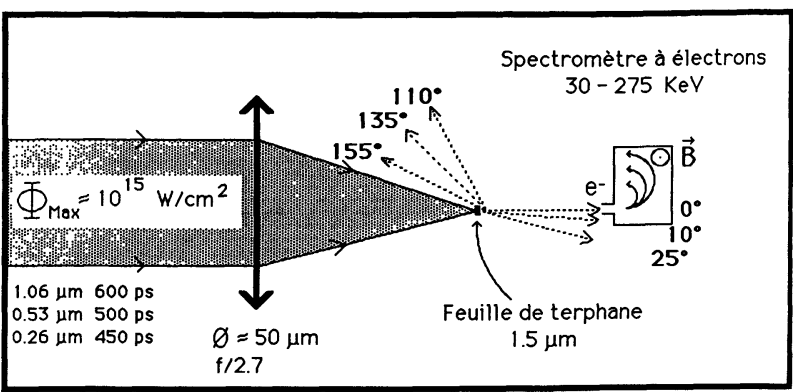

Fig. 1. - Représentation schématique de l'expérience.

[Experimental set up.]

du GRECO-ILM à l'Ecole Polytechnique délivre $100 \mathrm{~J}$ sur une impulsion de $600 \mathrm{ps}$ à mi-hauteur à $\lambda=1,06 \mu \mathrm{m}$; ceci représente un flux sur la cible de $2 \times 10^{15} \mathrm{~W} / \mathrm{cm}^{2}$; par doublage et quadruplage de la fréquence, nous disposons respectivement de $60 \mathrm{~J} / 500$ ps et de $30 \mathrm{~J} / 450$ ps soit $1,2 \times 10^{15} \mathrm{~W} / \mathrm{cm}^{2}$ et $8 \times 10^{14} \mathrm{~W} / \mathrm{cm}^{2}$. Le spectromètre à électrons présente une ouverture d'angle solide de $10^{-3}$ str. Il est constitué par deux plaques aimantées souples qui produisent entre elles un champ normal uniforme de 320 Gauss. Six détecteurs à silicium représentant six canaux d'énergie recueillent les électrons déviés dans ce champ. Le spectre énergétique accessible s'étend de $30 \mathrm{keV}$ à $275 \mathrm{keV}$.

\section{Résultats expérimentaux.}

Nous avons voulu dans ces expériences caractériser la partie à haute énergie de la fonction de distribution électronique c'est-à-dire que nous avons mesuré le nombre d'électrons chauds à six valeurs d'énergie en fonction de leur angle d'émission. D'un tel spectre, nous déduisons une «température » électronique qui ne doit pas être comprise au sens collisionnel : c'est le mécanisme d'accélération qui produit cette distribution et non la thermalisation ultérieure de ces électrons entre eux; en effet, le temps de collision caractéristique est grand devant l'impulsion laser (faible densité d'électrons chauds) et d'autre part, une thermalisation rend la population électronique isotrope (c'est le contraire qui est observé). La « température » est calculée à partir de la pente de la fonction de distribution $f(E)$, laquelle est assimilée à haute énergie à une fonction en $\exp (-E / k T)$. Rappelons qu'à une et à trois dimensions, les maxwelliennes en énergie sont respectivement en $1 / \sqrt{E} \times \exp (-E / k T)$ et $\sqrt{E} \times \exp (-E / k T)$. Les températures estimées par ces trois fonctions sont proches: par exemple (cf. Fig. 2), tir 61280 : $T(\exp (-E / k T))=48 \mathrm{keV}$ alors que $T(1 \mathrm{dim})=$ $54 \mathrm{keV}$ et $T(3 \mathrm{dim})=43 \mathrm{keV}$.

Dans les figures suivantes, $f(E)$ est le nombre d'électrons émis par le plasma par $\mathrm{keV}$ et par stéradian.

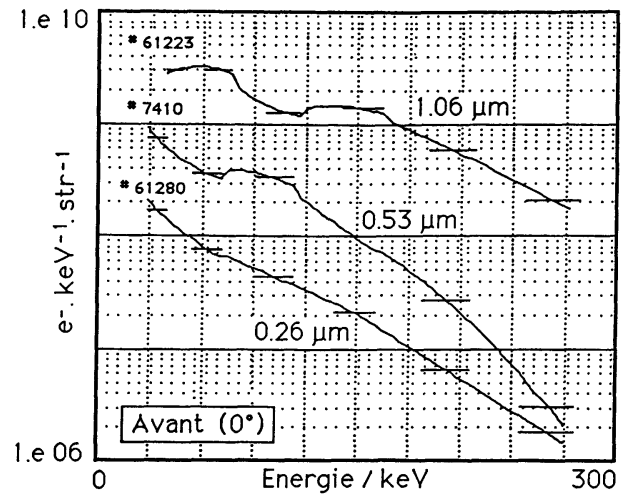

Fig. 2. - Spectres d'énergie à $\lambda=1,06 \mu \mathrm{m}, \lambda=0,53 \mu \mathrm{m}$ et $\lambda=0,26 \mu \mathrm{m}$ sur cible de terphane $1,5 \mu \mathrm{m}$ à l'avant $\left(0^{\circ}\right)$ $\left(\phi \approx 10^{15} \mathrm{~W} / \mathrm{cm}^{2}\right)$.

[Forward electron energy spectra at $\lambda=1.06 \mu \mathrm{m}$, $\lambda=0.53 \mu \mathrm{m}$ and $\lambda=0.26 \mu \mathrm{m}$ (terphane $1.5 \mu \mathrm{m}, \theta=0^{\circ}$, $\left.\phi \approx 10^{15} \mathrm{~W} / \mathrm{cm}^{2}\right)$.]

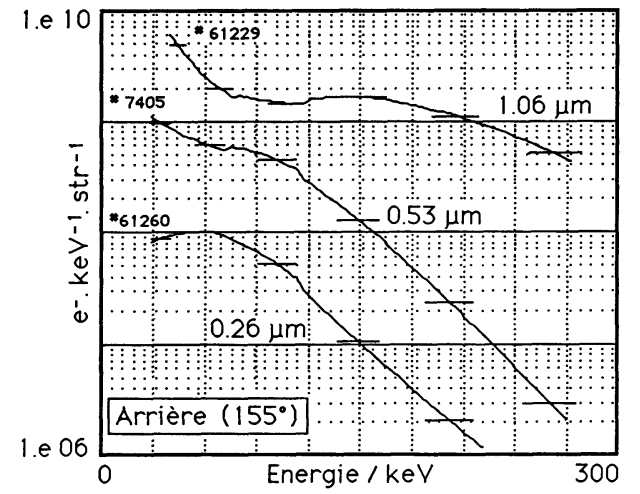

Fig. 3. - Spectres d'énergie à $\lambda=1,06 \mu \mathrm{m}, \lambda=0,53 \mu \mathrm{m}$ et $\lambda=0,26 \mu \mathrm{m}$ sur cible de terphane $1,5 \mu \mathrm{m}$ à l'arrière $\left(155^{\circ}\right)\left(\phi \approx 10^{15} \mathrm{~W} / \mathrm{cm}^{2}\right)$.

[Backward electron energy spectra at $\lambda=1.06 \mu \mathrm{m}$, $\lambda=0.53 \mu \mathrm{m}$ and $\lambda=0.26 \mu \mathrm{m} \quad$ (terphane $1.5 \mu \mathrm{m}$, $\left.\theta=155^{\circ}, \phi \approx 10^{15} \mathrm{~W} / \mathrm{cm}^{2}\right)$.]

En figures 2 et 3 sont représentés trois spectres typiques respectivement dans la direction du faisceau laser $\left(0^{\circ}\right)$ et à l'arrière $\left(155^{\circ}\right)$ aux longueurs d'onde laser $1,06 \mu \mathrm{m}, 0,53 \mu \mathrm{m}$ et $0,26 \mu \mathrm{m}$ (terphane $1,5 \mu \mathrm{m})$. Rappelons qu'à $0,53 \mu \mathrm{m}$ et à $0,26 \mu \mathrm{m}$, les flux maxima sont respectivement 2 et 3 fois plus faibles qu'à $1,06 \mu \mathrm{m}$. La température semble plus élevée à $1,06 \mu \mathrm{m}$.

La figure 4 présente trois spectres à $\lambda=0,26 \mu \mathrm{m}$ aux angles $0^{\circ}, 10^{\circ}$ et $25^{\circ}$ (terphane $1,5 \mu \mathrm{m}$ ). L'influence de l'angle d'émission des électrons sur leur «température » y est très sensible.

En figure 5 se trouve le diagramme angulaire de la « température » électronique, à $\lambda=0,26 \mu \mathrm{m}$ sur terphane $1,5 \mu \mathrm{m}$ au flux $5 \times 10^{14} \mathrm{~W} / \mathrm{cm}^{2}$. Ces «températures» sont de l'ordre de 30 à $50 \mathrm{keV}$ à l'avant $\left(0^{\circ}\right)$ et à l'arrière $\left(155^{\circ}\right)$. 


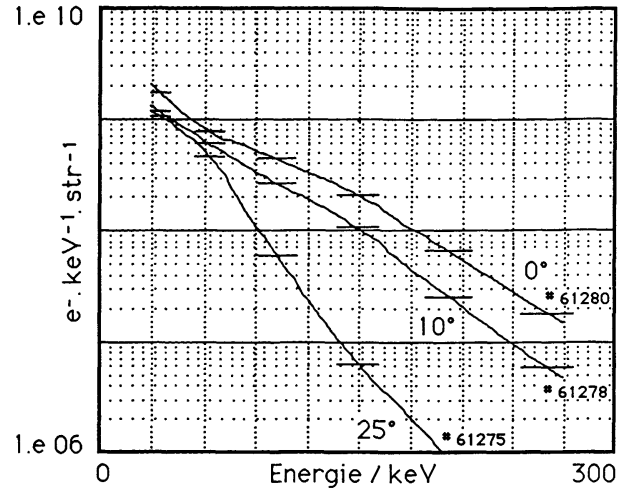

Fig. 4. - Spectres d'énergie à $\lambda=0,26 \mu \mathrm{m}$ aux angles d'émission $0^{\circ}, 10^{\circ}$ et $25^{\circ}$ sur cible de terphane $1,5 \mu \mathrm{m}$ $\left(\phi \approx 6 \times 10^{14} \mathrm{~W} / \mathrm{cm}^{2}\right)$.

[Electron energy spectra at $0^{\circ}, 10^{\circ}$ and $25^{\circ}$ from the target normal $(\lambda=0.26 \mu \mathrm{m}$, terphane $1.5 \mu \mathrm{m}, \phi \approx 6 \times$ $\left.10^{14} \mathrm{~W} / \mathrm{cm}^{2}\right)$.]

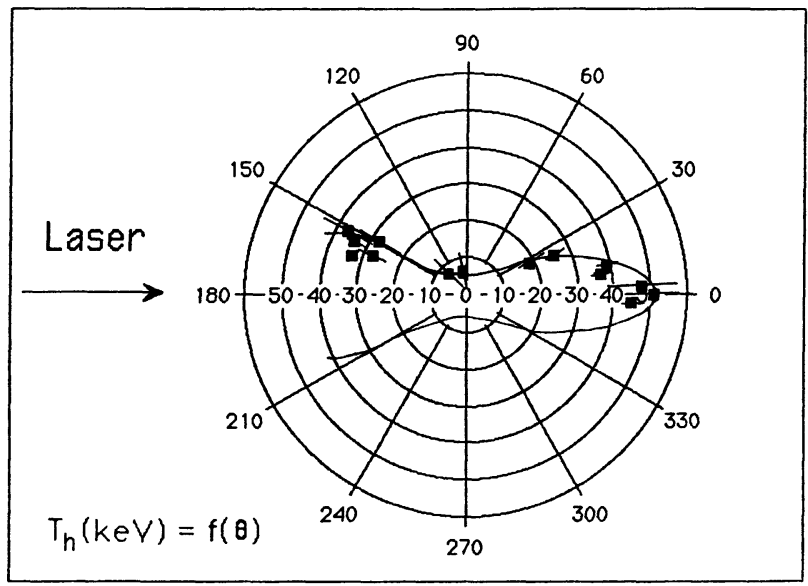

Fig. 5. - Diagramme polaire de la «température» électronique à $\lambda=0,26 \mu \mathrm{m}\left(\phi \approx 6 \times 10^{14} \mathrm{~W} / \mathrm{cm}^{2}\right)$.

[Polar diagram of hot electron «temperature» at $\lambda=0.26 \mu \mathrm{m}, \phi \approx 6 \times 10^{14} \mathrm{~W} / \mathrm{cm}^{2}$.]

Parallèlement sont dessinés figures 6 et 7 les diagrammes angulaires du nombre d'électrons chauds recueillis sur les différents canaux $(\lambda=$ $0,26 \mu \mathrm{m}$, terphane $1,5 \mu \mathrm{m}$, flux $\left.5 \times 10^{14} \mathrm{~W} / \mathrm{cm}^{2}\right)$. Le trait caractéristique le plus remarquable de ces mesures est le «piquage » du flux d'électrons vers l'avant d'autant plus accentué que leurs énergies sont élevées.

Notons enfin que d'après des mesures à flux variable à $\lambda=1,06 \mu \mathrm{m}$ sur cible de plastique $\mathrm{CH}$ d'épaisseur $75 \mu \mathrm{m}$, la «température » à l'arrière $\left(155^{\circ}\right)$ croît de $30 \mathrm{keV}$ à $\phi=2 \times 10^{14} \mathrm{~W} / \mathrm{cm}^{2}$ jusqu'à $70 \mathrm{keV}$ environ à $\phi=2 \times 10^{15} \mathrm{~W} / \mathrm{cm}^{2}$.

\section{Interprétation.}

La présence d'électrons énergétiques implique l'existence de champs électrostatiques intenses. La diffu-

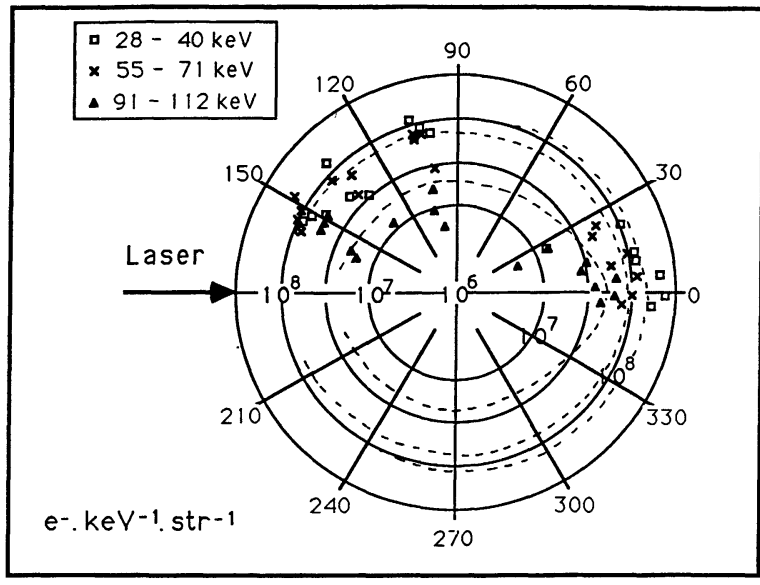

Fig. 6. - Représentation angulaire du nombre d'électrons chauds émis dans l'interaction à $\lambda=0,26 \mu \mathrm{m}$ sur terphane $1,5 \mu \mathrm{m}(28-112 \mathrm{keV})\left(\phi \approx 6 \times 10^{14} \mathrm{~W} / \mathrm{cm}^{2}\right)$.

[Polar diagram of hot electron emission $(28-112 \mathrm{keV})$ $\left(\lambda=0.26 \mu \mathrm{m}\right.$, terphane $\left.1.5 \mu \mathrm{m}, \phi \approx 6 \times 10^{14} \mathrm{~W} / \mathrm{cm}^{2}\right)$.]

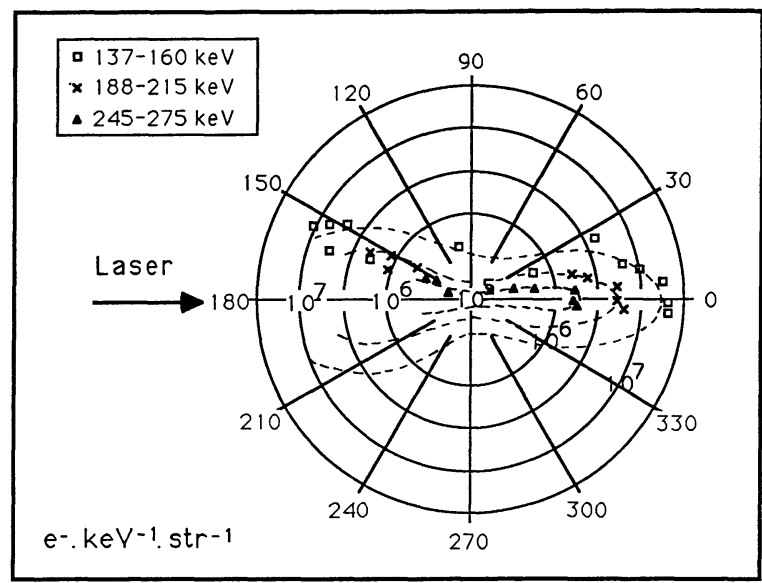

Fig. 7. - Représentation angulaire du nombre d'électrons chauds émis dans l'interaction à $\lambda=0,26 \mu \mathrm{m}$ sur terphane $1,5 \mu \mathrm{m}(137-275 \mathrm{keV})\left(\phi \approx 6 \times 10^{14} \mathrm{~W} / \mathrm{cm}^{2}\right)$.

[Polar diagram of hot electron emission (137-275 keV) $\left(\lambda=0.26 \mu \mathrm{m}\right.$, terphane $\left.1.5 \mu \mathrm{m}, \phi \approx 6 \times 10^{14} \mathrm{~W} / \mathrm{cm}^{2}\right)$.]

sion Raman stimulée et la décomposition deux plasmons sont des instabilités qui génèrent de tels champs. Toutefois, en ce qui concerne les électrons émis vers l'avant, la géométrie du deux plasmons ne favorise pas particulièrement la distribution électronique anisotrope observée et d'autre part, des mesures de corrélation entre le taux de Raman et le nombre d'électrons [4] émis rendent ce dernier mécanisme le plus probable.

La diffusion Raman produit une onde de plasma dirigée vers l'avant le long du laser incident, pour le Raman avant et arrière (Fig. 8). Dans le cas de diffusion avec un autre angle - Raman latéral - le vecteur d'onde plasma $\mathbf{k}_{\mathrm{p}}$ est toujours contenu dans un cône d'axe $\mathbf{k}_{0}$ dont l'ouverture augmente quand la 


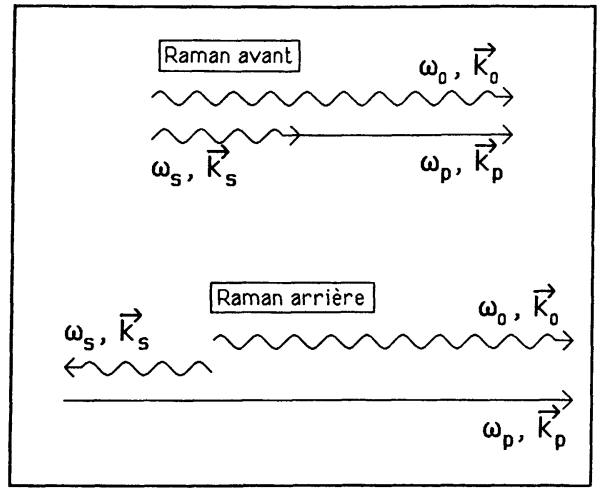

Fig. 8. - Géométrie du Raman avant et arrière.

[Forward and backward Raman scattering geometry.]

densité du plasma diminue ; par exemple, ce demiangle vaut $50^{\circ}$ pour une densité de $n / n_{\mathrm{c}}=0,05$, et vaut $90^{\circ}$ à la limite de densité nulle ; $n_{\mathrm{c}}$ est la densité au-delà de laquelle le laser ne peut plus se propager dans le plasma. Rappelons que l'instabilité Raman se déclenche jusqu'à $n_{\mathrm{c}} / 4 \quad\left(n_{\mathrm{c}}\left(\mathrm{e}^{-} / \mathrm{cm}^{3}\right)=1,1 \times\right.$ $\left.10^{21} / \lambda^{2}(\mu \mathrm{m})\right)$.

Dans un plasma froid où $k_{\mathrm{p}} \lambda_{\mathrm{d}} \ll 1$, il est facile, à partir des équations de dispersion et de conservation des ondes électromagnétiques et de la diffusion Raman, de calculer le vecteur d'onde $\mathbf{k}_{\mathrm{p}}$ de l'onde de plasma. Il apparaît alors que sa vitesse de phase $v_{\Phi}=\omega_{\mathrm{p}} / k_{\mathrm{p}}$ ne dépend que de $n / n_{\mathrm{c}}$. Si l'on considère que la «température » des électrons piégés dans cette onde est $T_{\mathrm{h}}=1 / 2 m v_{\Phi}^{2}$, sa mesure permet de remonter à la densité du plasma correspondante (cf. Figs. 9-11). Pour le Raman arrière, par exemple, dont le seuil est le moins élevé, des températures de 30 à $50 \mathrm{keV}$ correspondent à des densités de $0,19 n_{\mathrm{c}}$ à $0,23 n_{\mathrm{c}}$. Par contre, des électrons issus du Raman avant, dont le seuil est a priori atteint dans certains cas, ne sont pas forcément mesurables avec notre spectromètre : en effet, la vitesse de phase de l'onde de plasma associée au Raman avant est plus

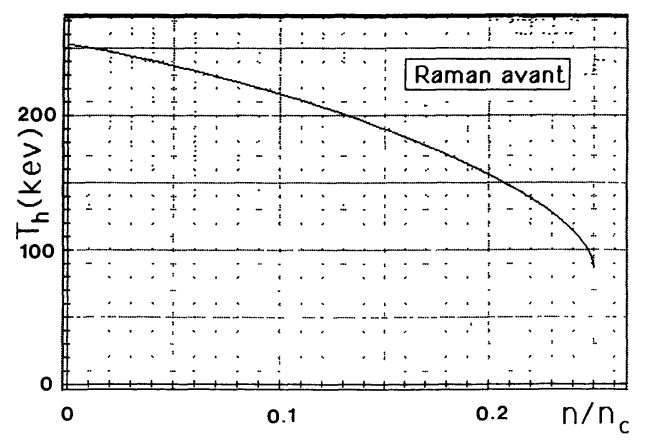

Fig. 9. - «Température » électronique associée au Raman avant.

[Hot electron «temperature » in Raman forward scattering.]

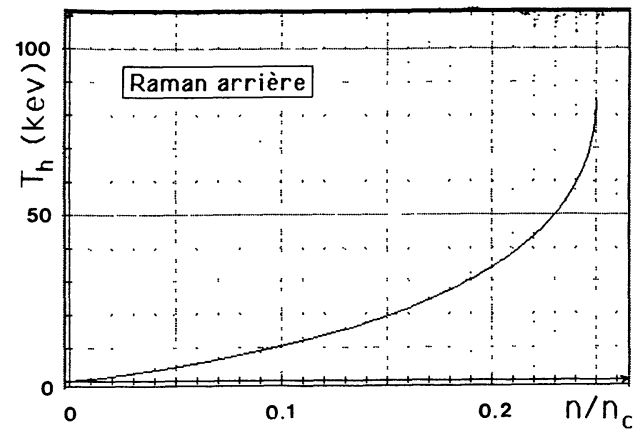

Fig. 10. - « Température » électronique associée au Raman arrière.

[Hot electron «temperature » in Raman backward scattering.]

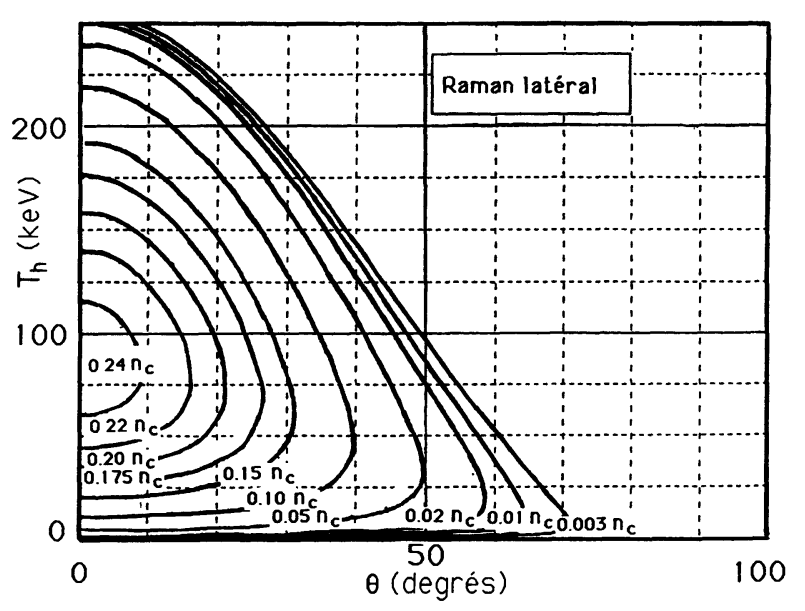

Fig. 11. - «Température " électronique associée au Raman latéral ; $\theta$ est l'angle entre la direction du laser et le vecteur d'onde de l'onde de plasma.

[Hot electron «temperature » in Raman side scattering; $\theta$ is the angle between the incident laser wave and the plasma wave vectors.]

grande que celle issue du Raman arrière et par conséquent le nombre d'électrons piégés dans cette onde en est plus petit. De plus, l'utilisation d'un spectromètre à gamme d'énergie plus élevée est nécessaire.

L'instabilité Raman ne peut pas par contre expliquer directement les électrons émis vers l'arrière (Figs. 6,7) ; remarquons qu'en plus le cône d'émission vers l'arrière semble plus large que vers l'avant. Ce phénomène peut être justifié par plusieurs hypothèses. Les ondes de plasma qui en sont responsables et qui peuvent provenir entre autres de la décomposition deux plasmons, se réfléchissent sur leur couche critique respective, phénomène d'autant plus probable qu'elles y sont créées à proximité. On peut aussi penser à la génération par effet Raman sur une structure de plasma plus complexe, par exemple inhomogène, présentant des trous de densi- 
tés, ou bien à la décomposition des ondes de plasma sur des fluctuations de densités ioniques.

\section{Conclusion.}

Ces expériences ont permis de dégager les points suivants : les électrons chauds issus de l'explosion d'une feuille mince à $\lambda=0,26 \mu \mathrm{m}$ sont caractérisés par une «température » de quelques dizaines de $\mathrm{keV}$; leur diagramme angulaire est dirigé préférentiellement selon l'axe du laser avec une largeur plus importante à l'arrière. Leur spectre d'énergie vers l'avant peut s'expliquer par l'accélération sur l'onde de plasma produite par le Raman arrière; par contre, l'émission électronique arrière est la manifestation de phénomènes plus complexes au sein du plasma qui méritent d'être étudiés avec soin. Quant à la détection électronique due au Raman avant, elle nécessite la mesure à plus haute énergie. Enfin, le calcul théorique de l'accélération d'électrons suppose la connaissance de la structure spatiale et temporelle du champ électrique. Il est en particulier difficile de quantifier le rôle du Raman latéral ou le rôle des électrons qui ne sont pas parfaitement cohérents spatialement et temporellement avec les ondes de plasma.

\section{Bibliographie}

[1] Tang, C. L., J. Appl. Phys. 37 (1966) 2945 ; Kruer, W. L., Phys. Fluids 23 (1980) 1273 ; Rosenbluth, M. N., Phys. Rev. Lett. 29 (1972) 565 ; Pesme, D., Laval, G., Pellat, R., Phys. Rev. Lett. 31 (1973) 203 ;

Forslund, D. W., Kindel, J. M., Lindman, E. M., Phys. Fluids 18 (1975) 1002.

[2] Liu, C. S., Rosenbluth, M. N., White, R. B., Phys. Fluids 17 (1974) 1211 ;

Kruer, W. L., Estabrook, K., Lasinski, B. F., Langdon, A. B., Phys. Fluids 23 (1980) 1326 ; 26 (1983) 1892.
[3] Liu, C. S., Rosenbluth, M. N., Phys. Fluids 19 (1976) 967 ;

Simon, A., Short, R. W., Williams, E. A., DewanDRE, T., Phys. Fluids 26 (1983) 3107.

[4] Labaune, C., Amiranoff, F., Fabre, E., Briand, F. (PMI, Ecole Polytechnique, Palaiseau), BAL DIS, H., VilleneuVE, D. (National Research Council, Ottawa, Canada), EsTabrooK, $\mathrm{K}$. (Lawrence Livermore Laboratory, California, USA), Rapp. d'activité du GRECO ILM 1985, Ecole Polytechnique, 91128 Palaiseau. 